\title{
Local Networking and Internetworking in the V-System
}

\author{
David R. Cheriton \\ Computer Science Department \\ Stanford University
}

\begin{abstract}
Local networking can be treated as a subset of internetworking for remote terminal access and file transfer. However, a distributed operating system, such as the V-System uses a local network more as an extended backplane than a fast, minature long-haul network.

This paper describes the use of server-based "intelligent gateways" to provide internetworking using standard protocols in conjunction with an efficient light-weight protocol for V IPC on a local network. Besides providing good local network performance. this design allows a gateway to act as an access control mechanism, addressing some reliability and security issues that arise with local networks.
\end{abstract}

\section{Introduction}

A local network can be viewed as an extended backplane when it interconnects a cluster of the workstations and server machines managed by a distributed operating system. The cluster operates as a multi-user system with the distributed operating system providing services analogous to a conventional multi-user machine and operating system.

The V-System ${ }^{1}$ (pronounced "Vee") is a distributed operating system developed at Stanford that uses Ethernet ${ }^{2}$ as such an extended backplane to connect a collection of diskless SUN workstations ${ }^{3}$ and server machines. A distributed kernel provides uniform, transparent message-based interprocess communication on a single workstation and between processes on different workstations. Dedicated server processes, often running on dedicated server machines, provide file access, printing. internetwork access and other services. Application processes access system services using stub routines that format, send and receive messages to interact with the required servers.

We have developed a specialized light-weight local network protocol for interkernel communication between different workstations. We developed this protocol rather than using an existing protocol for two reasons. As part of our research, we are investigating specialized protocols to improve network IPC performance, which has been a major problem with previous attempts to realize the basic system design of V. (The $V$ design is similar to many message-based and distributed operating systems.) Atso, there is no established standard interprocess

This work was sponsored in part by the Defense Advanced Research Projects Agency under contracts MDA903-80-C-0102 and N00039-83K-0432.

Permission to copy without fee all or part of this matcrial is granted provided that the copies are not made or distributed for direct conmercial advantage, the ACM copyright notice and title of the publication and its date appear, and notice is given that copying is by permission of the Association of Computing Machinery. To copy otherwise, or to republish requires a fee and/or specific permission. communication protocol that suits the $\mathrm{V}$ interprocess communication. Nevertheless, we have a strong need and desire to support and use standard internetwork protocols, in particular, being part of the ARPA Internet community, the IP/TCP family of protocols.

Normally, use of internetwork protocols on a local network is not a problem. Most local networks are used for Remote Terminal Access and File Transfer (RTA/FT). similar to long-haul networks. For RTA/FT users accustomed to long-haul network performance, local network performance is more than adequate even though internetwork protocols may impose a significant overhead and end-to-end data rates may not be comparable to the underlying local network speed.

However, our use of a local network as an exlended backplane means that local network performance must be compared to intra-machine communication performance, not long-haul network performance. Using this comparison, we argue that using internetwork protocols on a local network for IPC communication leads to minimal benefits and significant costs.

This paper describes the use of server-based "intelligent gateways" to provide internetworking using standard protocols in conjunction with an efficient light-weight protocol for V IPC on a local network. Besides providing good local network performance, this design allows the gateways to efficiently implement access control mechanisms, addressing reliability and security issues that arise with local networks.

The next section describes local networks, particularly the problems they pose for computer commiunication. The following section explores the issues in using internetwork protocols, in particular ARPA Internet protocols, as a basis for local network IPC. We then describe the V-System interprocess communication and its performance on a local network. Subsequent sections describe our internetworking techniques.

\section{Local Networks}

By local network, we mean a multi-megabih, low delay, low error rate communication facility connecting up to a few hundred computer nodes over a few kilometers at relatively low cost. The Cambridge ring ${ }^{4}$. MIT ring ${ }^{5}$ and Xerox Ethernet ${ }^{2}$ are a few examples.

The advantages of local networks need no repeating here. Suffice it to say that they provide sufficiently high bit transfer rates between nodes to makes peripheral sharing (e.g. disk storage) and truly distributed computation practical. There are, however, several problems to solve before realizing this potential.

\section{Efficiency}

It is difficult to achieve data transfer rates anywhere close to the bit transfer rate of most local networks with current protocols and interfaces. For instance, the maximum data transfer me of a Telnet connection between two VAX/UNIX systems over the 3 megabit Ethernet at Stanford is about 56000 bits per second. about 2 percent of the raw network speed. 
In measurements of data transfer lime between two SUN workstations ${ }^{3}$, Motorola 68000 -based workstations running at $8-10 \mathrm{MHz}$, using no protocol ${ }^{1}$, the time to transfer 512 bytes between $8-\mathrm{MHz}$ workstations connected by 3 mcgabit Ethernet is 3.65 milliseconds, almost three times the 1.39 milliseconds expected from the bit transfer rate of the network. Performance is worse relative to physical bit rate using a faster network (such as the 10 megabit lithernet) and adding the necessary protocol processing for reliable data transport.

These measurements clearly indicate that, unlike long-haul networks where physical communication capacity is usually the critical resource, processing and interface overhead is critical in local network performance. Therefore, local network protocols should be designed to minimize processing overhead, presumably exploiting the low error rate and low delay of local networks. Similariy, network interfaces should oflload the processor in some cost-cffective fashion. While DMA is the obvious approach to fast network interfaces, without some type of scatter-gather capability as part of the interface the processor is generally forced to do an extra copy of each packet.

Several studies have shown that the chnice of protocol and its implementation must be carefully tuned to local network characteristics to achieve performance commensurate with local network speeds. Spcedups by factors of up to 30 have been reported as a result of stripping away layers, honing the protocols and tuning the implementations ${ }^{6,7}$. In most cases, processor time used by a protocol is the crucial issue in performance, not the network bandwidth. This is certainly the case with the $V$ interkernel protocol ${ }^{\mathrm{l}}$.

\section{Reliability}

The speed of a local network presents a reliability problem for all connected machines because one machine can flood one or more others with packets faster than the packets can be discarded. For instance, a workstation that fails in a tight loop sending erroneous packets to a network file server can easily generate packets faster than the lile server can discard them. This will dramatically degrade, if not disable, the file server for properly behaved clients.

Another similar failure is what might be called broadblasting, where a node sends out broadcast packets very rapidly. If, as is tvpical on the Ethernet, every node receives all broadcast packets, packets are handled at the kernel interrupl level, and broadcast packets are generaled faster than they can be processed, every machine on the network is effectively disabled.

Over the past 2 years, the author has observed several such failures on the Stanford 3 megabit lithernet which currently connects about 100 machines. These failures are notable in both the number of machines affected and the amount of difficulty in tracking down the problem, with machines in obscure locations behind locked doors. Besides total failure, we have also observed unexplained load and significant response degradation that were subsegulutitly traced to a high level of strange network activity.

This problem is not unique to the Fthernet but could be cxpected on any local network not explicitly (and expensively) engineered to prevent it.

In contrast, dealing with bad packets is not such a problem in long-haul networks because of their low speed and restricted user-level access to the network. Established techniques for authentication, error checking and access control suffice to protect each host.

\section{Security}

Most local network tcchnologies are based on a common shared channel so all host-network connections "sce" all the data When data is sent unencrypted, it is generally possible for each host to read all data that is transmitted. Encryption adds a significant overhead, not only for the actual encryption process but also for the extra communication required for distribution of encryption keys and authentication 8 .

A nore subtle problem arises from the use of broadcast to locate a server. An impostor can respond and play havoc with the requesting client. We have expcrienced this problem at Stanford, not from malicious intent, but from someone bringing up their own version of a name server which responded incorrectly. The name server ran on a lightly loaded machine. invariably responded first and effectively disabled the name service by responding incorrectly. This problem might also be considered an aspect of the reliability problem when it arises from failure as opposed to malicious intent.

\section{Summary}

These threc problems can be addressed by hardware (or hardware-supported) mechanisms in every node. For instance, a large host can feasibly have a front-end communications processor to insulate it from the broadblasting and packet flooding problems. However, the V-System, as with many distributed operating systems, is designed for modest-sized workstations and server machines. In our design, the protection and security mechanism is concentrated in the gateway, thus amortizing the cost over multiple workstations. Local communication avoids extra overhead for reliability and security by relying on the gateway. Misbchavior on the local nctwork can be deiected by networking monitoring programs that take advantage of the "shared channel" structure of most networks, e.g. promiscuous mode on the Ethernet.

In the next section, we argue that internetwork protocols do not provide the best basis for solving these problems.

\section{Internetwork Protocols}

Standard internetwork protocols can be used on local networks, treating each local network as just another network in an internetwork. For example, the ARPA Internet protocols 9 provide a uniform base of internetwork packets (IP) ${ }^{10}$ across both long haul and local networks independent of network lechnology. A transport-level protocol, $7 \mathrm{CP}^{\mathrm{H}} 1$ provides bi-directional byte streams for internetwork RT $/ \mathrm{FT}$. When a local network is used primarily for RTA/FI, this is a simple and effective approach. For instance, the Stanford Ithernet-based campus network has run in this mode for several years using the NRPA Internet and PUP family 12 of protocols.

However, internetwork protocols do not provide a communication model wcll-suited to distributed systems. A distributcd system requires a transport-level facility for interprogram and possibly intra-program communication that works transparently within a single machine as well as across the network between machines. Because of the predominant use of procedure call-like communication within systenis (even messagebased systems) the appropriate modei is some form of requestresponse transaction-style communication, where a transaction typically consists of requesting a service and gctting a response back. With appropriate language processor support, this can take the form of a tiansparent remote procedure call $(R P C)$ as an extension of the procedure call mechanism of the programming language system, as was done for Mesa ${ }^{6}$.

The Internet protocols present two options for implementing this communication: TCP byte streams or IP datagrams. 


\section{TCP Byte Streams}

TCP, the transport-level of the Internet protocol family, provides a bi-directional byte stream. This is preciscly the right choice for internetworking because it supports R'l'A/FT well and allows efficient implementation over long-haul notworks using standard techniques like sliding window flow control and piggybacked acknowledgements. However, for distributed system communication on local networks, byte streams provide little benefit to RPC-like communication and impose significant overhead to implement. Consider, for instance, a file write operation across the network.

In general, a network datagram can hold file parameters plus a file page or block, Using a low-level specialized protocol, a network file write consists of sending a packet containing data and parameters to the file server and recciving a reply packet back, with reliable transport implemented relying on network CRC checks and retransmissions until a valid reply packet is received. For added efficiency, the specialized protocol can rely on the idempotency of the write operation and not bother to filter out duplicate packets arising from retransmissions or to save reply packcts for retransmission.

Using a byte stream, a copy of the data is made on transmission to allow for retransmission even though the process will typically wait for a reply message. Similarly, a copy is made of the reply even though the operation may have been idempotent. Furthermore, a bytc stream-level acknowledge is sent for the request data in addition to the IPC-level reply that is set to provide return values for the operation. The IPC mechanism must also impose a message structure, similar to the datagram structure, on top of the byte streams, thus contributing to further overhead. Also, the IP and TCP headers add 60 bytes to every packet. Finally, the byte-stream model imposes a cost for connection setup and tear-down, which becomes significant if a separate connection is established for each message transaction and otherwise requires a sophisticated connection management mechanism.

According to our measurements, a V kernel communication mechanism implemented using. TCP would be slower by more than a factor of 2 and signilicantly complicate and enlarge the kerncl code. The slowitown is primarily due to the extra processing overhead because roughly the same number of packets are sent in both cases.

With the natural realization of request-response communication using datagrams, an alternative is to implement a spccialized rcquest-response transport-level protocol using IP, the internctwork packet protocol or UDP, the user datagram protocol, as a basis. While we do not preclude this approach in our work, (in fact there is an IP. based version of the V kernel), there are costs associated with this approach and fewer benefits than one night expect, as described below.

\section{Internet Datagrams} to:

Encapsulating all packets in IP packets introduces overhead

1. Construct and prepend the IP header along with transmilting and recciving the header.

2. Calculate the checksum(s).

3. Determine the internetwork destination address as well as the local network address of the next (and usually only) hop.

Using UDP, one may incur the additional overhead of calculating a checksum for the data portion of the packet. Note that we are only considcring the case of the packet going to a destination on the same local network so fragmentation and reassembly are not an issue.

Using an IP-based version of the $V$ kernel using no checksums and no packet routing or reassembly (i.e. only constructing and prepending the IP header), we measured a slow-down of 20 percent for 32 -byte data mossages (the common size used in our system). While larger messages such as those for transporting file pages incur less percentage overhead. the addition of IP and UDP checksums, intcrnctwork routing and reassembly would increase the overhead. The significant cost in space for code and data for packet reassembly and addressing should also be considered.

Note that it is important not to overestimate the cost of general protocols. Clark 13,14 has shown that the overhead can be minimized by careful design, choice of algorithms and optimization for the common case. Even reassembly can be done in roughly 400 instructions of code with an overhead of one or two tests in the normal case of one-fragment packets.

However, there are other costs to encapsulating local communication in IP packets. For one, broadcast and multicast IP packets present problems. We are exploring the use of multicast for efficient communication in a distributed computation. We use a publishing paradigm in which information is distributed by a publisher to multiple subscribers using multicast packets. IP docs not define behavior for broadcast and multicast packets. In fact, because it is not feasible or necessarily desireable to support multicast across an internetwork, it is unclear how to define this facility without violating the transparent, uniform internetwork behavior of IP.

Using a purcly local network protocol, the domain of broadcast or multicast is automatically defined to be a single local network, and the underlying hardware generally supports this facility directly. Moreover, with the local network protocol implemented directly on top of the network interface provided by the hardware, easier access is provided to the multicast filtering implemented by some network interfaces.

Our experiments with an IP-based version of the $\mathrm{V}$ kernel pointed out another problem wilh this approach. We discovered our IP gateway to the ARPAnet goes into a loop retransmitting any broadcast IP packet it sees until the hop count in the packet exceeds the maximum hop count. This gateway was developed outside of Stanford, we cannot remedy the problem ourselves, and we are told that the problem is non-trivial to fix because of the design of the gateway. The nct result is that we currently cannot run our IP-based version of the kerncl with the IP gateway operational. The problem results in part because IP does not define behavior for broadcast packets. Whilc one might argue that this problem will be eliminated with a fix to the gateway, we contend that it is indicative of the type of problems that occur when internetwork mechanisms are exposed to unusual communication peculiar to local networks.

Another cost of IP encapsulation is the loss of meaning to the packet type ficld provided by most networks. Local network datagrams typically contain a type field specifying the type of protocol used with each datagram. If a local nctwork protocol is so identified, an IP gateway can discard such packets at the lowest level with minimal overhead. If all communication is encapsulated in IP packets, this type field becomes meaningless and higher-level software is required to demultiplex or discard packets. This leads to a confusion of layers in the IP-based version of the $V$ kernel where the kernel must intercept IP packets containing $V$ interkernel packets but passes other packets, both IP and non-1P, through for access by process-level implementations of other protocols like PUP BSP and ICP.

These overieads need to be compared with the benefits of using IP encapsulation. Superficialiy, it wotid appear that IP encapsulation allows one to transparently extend the network IPC across gateways. However, several problems arise. First, IPC messages are addressed to processcs. not machines, so process naming musi also be extended to mulliple networks. The larger and more general nams space required as a conscquence has a detrimental effect on intra-macine and boal inctwork IPC performance.

Second, the timeout periods and maximum retransmission 
count that are tuned for local network specd, detay and error rate are unsuitable when additional delay and error is introduced by the gateway. For instance, a kernel may retmintmit and timeout before a gateway has managed to transmit the first packet on a slow connected network.

Finally, we have argued earlier from a reliability and security standpoint that a gateway should act as an access control mechanism. However, it is relatively difficult to implement efficient access control in an Internet gateway because it is intended to act as a simple packet shiuffer, performing only routing and fragmentation/reassembly. This is in fact one of the intentions behind using internetwork datagrams.

In general, IP offers uniform transparent communication across the internetwork in the form of datagrams, as a generalization of the datagrams in a single packet-switched network. Distributed computation seens best supported by a uniform, transparent communication mechanism that is an extended implementation of an intra-machine communication mechanism. The uniformity and transparency of distributed system communication cannot be extended fully to the domain of IP packets simply by encapsulating messages in IP packets. Moreover, the degree to which it does extend is difficult to define or control. In addition, IP introduces a significant performance penalty.

Considering that 95 percent of the communication is local in our system, these arguments show that local network IPC protocols are best separated from internetwork protocols. In many ways, sending local IPC in IP packets seems analogous in overhead and potential security/reliability risk to sending office memos around the office in airmail envelones. Although our discussion has focused on the IP/TCP protocols, our conclusions appear to apply to other internetwork protocol families such as XNS and the X.25/X.75 family.

\section{Two Fallacies}

The author has been exposed to two other arguments against using local network protocols in place of internetwork protocois that are misleading if not incorrect.

Argument 1: If local networks do not use internetwork standard protocols, cvery local nctwork will use a different set of protocols.

Counter Argument: There can be a standard for local networks that is distinct from internetwork standards (the latter are then used primarily between networks). Consider as an analogy the electrical power distribution system. There are standards for wide-area distribution using voltages of 50,000 Volts and up, well suited to long distance transmission. However, the local standard of 120 Volts is not forced by any link to the wide-area standard but by the economies of standardization, e.g. standard appliances with standard plugs. Gateways can act analogously to the transformers in.this analogy, converting between internetwork protocols and local network protocols. Using internetwork protocols locally would be like having a 50,000 Volt service in one's housc and consequently require, for instance, a 50,000 Volt toaster.

Argument 2: Protocol translation is impractical because one ends up with $\mathrm{N}$ protocols and $\mathrm{N} \times \mathrm{N}-1$ protocol translators. Morcover, it is never possible to exactly translate from one protocol to another.

Counter Argument: This argument is true if $\mathrm{N}$ is large and the protocols are relatively high-level or diverse. However, only one local nctwork transport protocol is required, as with the VSystern. It is only necessary to translate between it and usually one internetwork protocol. Becausc the local network mechanism is used for all inter-program communication and the clicnt-server interface uses this inter-program communication, the translation occurs as part of a client accessing the server and the server implementing the internetwork transport protocol under the control of its client interface. For example, because the local V IPC is used for inter-program communication, any program that implements (for instance) TCP automatically translates between the local IPC to TCP because the local IPC is its "user interface". Thus, there is one protocol translator for each internctwork protocol, namely the implementation of that internetwork protocol. The predominate use of internetwork communication for RTA/Fl (including mail) further simplifies the problem. Finally, higher-level internetwork communication can be handled by special programs. For instance, the details of a particular virtual terminal protocol can be buried in a program implementing remote terminal access.

In general, the internetwork environment and internetwork protocols provide a very powerful communication facility. The challenge is to provide access to this without compromising the potential of local network communication. The next sections describe the approach taken in V.

\section{Local Network Communication}

All transport-level local network communication in the VSystem uses the message-based interprocess communication facility provided by the distributed $\mathrm{V}$ kernel. Interprocess communication is transparent with respect to the processes being on the same host or different hosts.

This facility provides an ideal base for a distributed system because it allows software above the kernel level to be designed and implemented independent of whether the clients it serves and the services it uses are local or distributed. For instance, we are using diskless personal workstations with all file access and program loading via IPC messages to network file servers. Adding a disk and local file server process to a workstation requires no changes to the workstation software other than adding a disk driver to the kernel.

In this use of a local network as an extended backplane, the vast majority of the traffic is local to the network. Consequently, $\mathrm{V}$ network IPC is implemented using a specialized local network protocol that takes advantage of transactional communication and the properties of local networks. The following briefly describes the IPC model provided, the protocol and its performance. For further details, the interested reader is referred to a separate in-depth study 1 .

The sending and receiving processes are specified by process identifiers, structured as two 16-bit subfields: logical host and local process identifier.

\section{\begin{tabular}{l|l} 
logical host & local process identifier
\end{tabular}}

Fig.1 Process Identifier Subfields

The logical host field is mapped to a particular network address and the local pid is mapped to a process on the machine at that nctwork address.

The basic interprocess communication is in terms of three primitives: Send, Receive and Reply that implement a message transaction or request-response pair of messages, as shown in fig. 2. 


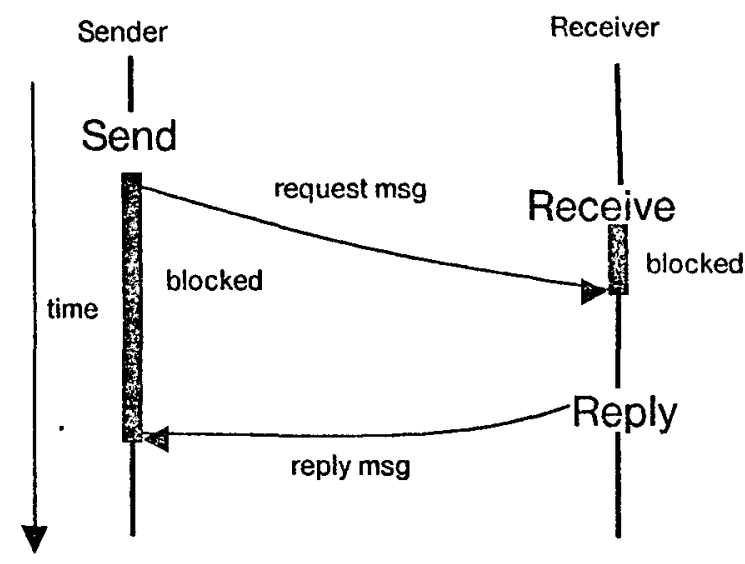

Fig. 2 Send-Receive-Reply Message Transaction

The sender sends a message to the receiver and is blocked until the message is received and it receives a reply message back. The time for a Send-Receive-Reply sequence using 32-byte messages between two processes on separate SUN workstations connected by a 3 megabit Ethernet is 2.56 milliseconds.

Messages are restricted to 32 bytes. Larger data transfers are handled by separate primitives, MoveTo and MoveFrom, which move an arbitrary number of bytes between the sender and the receiver of a message while the sender is still awaiting the reply message. Basically, the recipient of a message can use MoveFrom and MoveTo to read and write the memory space of the message sender up to the point that the reply message is sent.

Using MoveTo for program loading from a network file server into a disklcss SUN workstations (assuming the program text is in the file server's memory buffers), a 64 kilobyte program can be loaded in 338 milliseconds on the 3 megabit Ethernet. This performance is within 13 percent of the speed at which a SUN workstation can write packets out to the network with no protocol overhead. It is also a factor of 5 faster than the maximum data rate we can achieve using our TCP implementation over the Ethernet.

Streams can be implemented efficiently on this IPC facility as well. For instance, with a disk delivering a 512 byte page every 15 milliseconds, a file can be read sequentially averaging 17.13 milliseconds per page. This is comparable to the performance of highly tuned special-purpose fitc access protocols ${ }^{15}$. With this performance, the V IPC facility is also entirely adequate as a transport level for remote terminal access and file transfer.

The efficincy of the interprocess communication is in part due to the use of a request-response model of communication and matching protocol. For instance, the response serves as an acknowledge to the request as well as transporting a reply message. MoveTo and MoveFrom are implcmented as multiple packet versions of a request-response interchange. Buffering and flow control are made simple by the synchronous communication regime arising from request-response. Surprisingly, the requestresponse model still allows considerable concurrency of ex ecution between two communicating nodes despite the synchronism of the client interface. Performance also benefits from use of a minimal network header and direct mapping from process identifier to network address.

The efficiency of the V kernel IPC is essential to the practicality of this approach, yet requires the communication be specialized to local network operation. The following sections describe how we provide internetwoik communication with no penalty to local network performance.

\section{Logical Local Networks}

A logical local network is a collection of two or more physical local networks that logically function as one network from the standpoint of the node nctworking software. When multiple networks are required because some physical limitation of the networking technology has been exceeded (such as maximum number of nodes or maximum length), one can avoid changing existing networking software by organizing multiple physical nctworks as one logical network. This requires that the local network addressing be adcquate to address all the nodes of the logical network. For instance, the 48-bit Ethernet address is (theoretically) adequate to uniquely address cvery Ethernet port in the world. Also, if the different physical networks use different tcchnologies, the datalink packets must either be compatible or easily mappable from one form to another. Finally, it requires a connecting node we call a bridge that can recejve all packets addressed to nodes on other networks and retransmit these packets on the appropriate networks transparently with respect to both the sender and receiver of the packet. Generally, a bridge receives all packets transmitted on the networks they connect but only transfers the appropriately addressed packets to a connecting network.

For example, the Stanford research network includes several Ethernets connected by bridges. The bridge is built from a SUN workstation with Ethernet ports on two or more networks receiving promiscuously. It is basically a software version of the Ethernct repeater except it does not transmit packets destined for a node on the network of origin to the other networks. We measure a 2.7 millisecond delay in transmission through the bridge for a 1024 byte packet. V kerncl IPC is regularly used through one and two bridges, although with some degradation in performance. Thus, except for performance, multiple physical Ethernets appear as a single network to the networking software. In addition, "local" traftic on one physical network (except for broadcast) does not load other physical networks.

Using bridges, a single "logical" network could be quite large (into the thousands of nodes) and in fact use different network technologies (up to the limitations mentioned above). However, our experience has been that one soon encounters problems of security and reliability, not to mention addressing, packet format and packet size compatibility problems.

Although hardly a new idea, logical local networks are mentioned here to show that the use of multiple physical networks does not necessarily imply the need for internetworking in the conventional sense. In their limitations, they also point out the need for access control for large-scale networking.

\section{V-System Internetworking}

V IPC internetworking is supported by an intelligent gateway that provides local alias processes for remote processes. A local alias process is a (pscudo) process created by a $V$ interkernel gateway that represents on the local network a process that executes on a machine connected to another network, which we call a remote process. Fior a process to communicate with a remote process, it must first ask the gateway to create a local alias for the remote process, and then specify this local alias process in all IPC operations. $\Lambda \mathrm{n}$ agent in the gateway takes the necessary action to translate IPC with a local alias process into IPC with the remote process. 


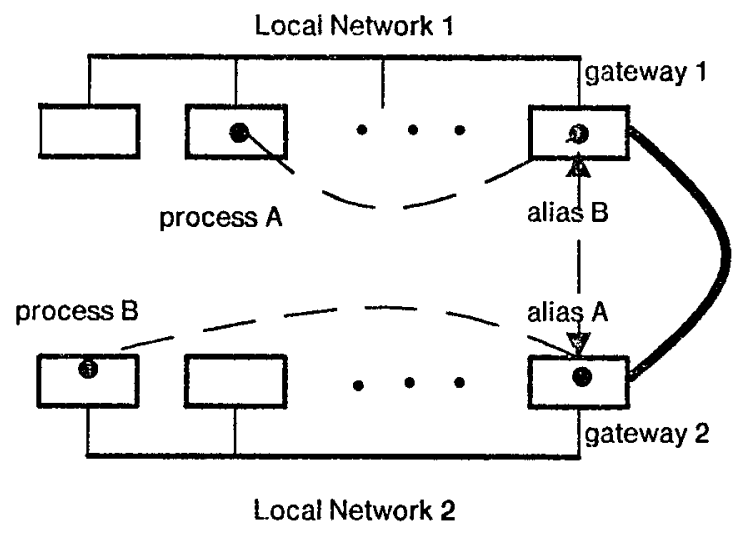

Fig. 3 Remote IPC using Alias Processes

In Fig. 3, process A sends to alias process B (transparently implemented in the local gateway) which communicates with the gateway on process B's network to cause the alias process A to send to process $B$. The message is sent by $A$ just as though $B$ is local to its network and received by $B$ just as though $A$ is local to its network.

A range of process identifiers are reserved for use as local alias process identifiers (the 16th bit of the process identifier is 1 for an alias process and 0 for a real process). This makes identification of alias processes easy for the kernel and for applications.

The use of local alias processes for internetworking has several advantages.

\section{No Penalty to Local Communication}

First, internetworking docs not impose any cost on local network communication. Local IPC takes place just as though internetworking had not been considered at all in the design. Packets addressed to alias processes on the gateway machine are simply passed by its kernel to the interkernel gateway software instead of being processed directly by the kernel as done for the local case. This structure has the additional advantage of proviaing a clean split between the handling of local network communication and internetwork communication.

\section{Internetwork Transparency}

Internetwork communication is almost identical to local network communication. A process can communicate with a remote process through a local alias as though they were on the same network, and in fact as though they were on the same machine.

A flaw in transparency arises because the process identifier name space is local to a logical local network, so process identifiers cannot be passed and used transparently between networks. A process identifier on network $l$ must be translated into an alias process identificr for network 2 before being passed to a process on network 2 . Otherwise, the process identifier would be interpreted incorrectly on network 2. In particular, when the file server responds to an open file request specifying the scrver process that will handle read and write operations on the file, it must be aware of whether the process is remote and perform the translation of the process identifier to a remote alias if so.

Problems arising from this complication are reduced by several considerations. First, the primary use of this facility is for accessing a remote file server, printer server or other standard server. It is not unreasonable for such servers to be properly progrimmed to handle the alias translatior. Second, in our experience to date, process identifiers are rarely included in messages. Third, for security, accounting and scheduling, it is often desircable for a server to know whether a process is local to the network or not. For example, a printer server may give higher priority to locally submitted print jobs. while still accepting remote submissions. Finally, tcsting for "remoteness" is efficient because it is indicated by a bit in the process identifier.

\section{Access Control}

Control over the creation of local alias processes allows the gateway to control access to a network bccause, without a loca! alias process, no communication is possible. Authorization can be sought from a local authentication server or from the designated receiving process. Moreover, local processes can easily identify alias processes, and thus remote processes, by examining the process identifier.

We imagine the gateway by default not allowing messages from outside the network to be sent to arbitrary local hosts. Servers designed to provide services via internetwork access explicitly authorize the gateway to provide this access. Because the required alias processes are created previous to communication occurring and may persist over the lifetime of the associated processes, access control does not impose an overhead on individual IPC operations.

In summary, the use of local alias processes allows internetwork communication to be provided with no local networking penalty, a strong degree of transparency, and a simple mechanism for controlling access to the network. The next section describes the basic gateway design that provides this capability.

\section{Gateway Design}

A V interkernel gateway connecting two V-System local networks is structured as two half gateways, with each halfgateway viewed as a protective "front door" for the network to which it is attached. This contrasts with the conventional motivation for half-gateways, namely reducing the amount of different galeway sofiware required (although we plan to benefit from this advantage too). Half-gateways are also appealing because the administration of cach network can provide and maintain its respective half. In general, while internctworks are generally drawn as networks connected by gateways, the protection, accounting, security and administration boundary runs through the gateway, as illustrated in Fig. 4.

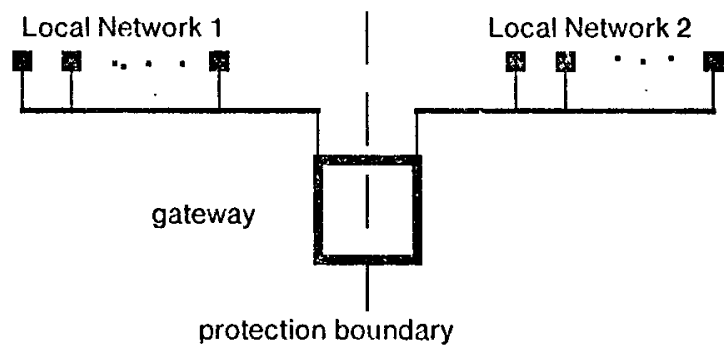

Fig. 4 Internetwork Bounclary Splitting a Gateway

Each half gateway runs on a scparate processor, communicating with the other half by a large shared memory buffer area, as shown in Fig. 5. 
Net 1

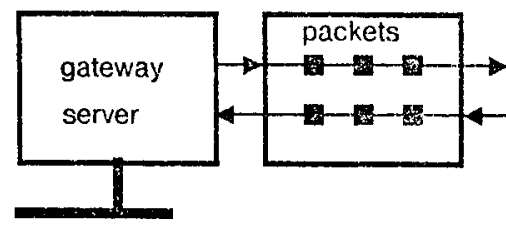

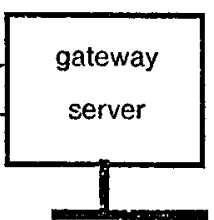

Net 2
Fig. 5 Dual-Processor Gateway Structure

The use of separatc processors and loosely coupled communication between them prevents either nctwork from totally disabling the gateway by bombarding the gateway with packets. The bombarded half may be effectively disabled in handling the packets but the other half can continue to function. In particular, it can take some action in response to the packet flooding from the other half. Using separate processors also has the advantage of insulating the processor rcquirements of one half of the gateway from the other. Finally, each network can use extra cycles in its half-gateway processor for running additional software, an authentication and name server being one good example. In fact, because the gateway uses a standard version of the $\mathrm{V}$ kcrnel, we can run such a server on the half-gateway transparent to the gateway software, the clients of the server and the server itself.

The gateway software itself is structured very similarly to the kernel software. In fact, the current gatcway software is a version of the kernel heavily modified to run as a process and to implement alias processes and the required communication with remote networks. The real kernel simply passes $\mathrm{V}$ interkernel packets addressed to alias processes on to the gateway software, rather than handling them itself, as it does for real local processes.

Gateways take an active role in optimizing communication with remote processes across the long-haul connections. For instance, suppose two networks are only connected by a long-haul network that uses TCP. In this case, the two half gateways impiementing the alias processes are also separated by an Internet TCP connection. Because TCP provides a reliable transport connection, the retransmissions generated as part of the local IPC transport mechanism can be filtered out and "ack'ed" by the gateway, eliminating unnecessary traffic over the long-haul connection and insulating the time-out for local network operation from the delays of long-haul communication.

As a further optimization the gateway can combine IPC packets as appropriate to make the optimum use of the TCP connection when IPC packet boundaries are not significant. For instance, a MoveTo data transfer consisting of multiple packets can be transformed into a TCP bytes stream with the packet boundaries discarded. The receiving gateway is free to repacketize the byte stream into IPC packets of a size most suited to its network. The lack of significance to packet boundaries in $V$ IPC bulk dala transfers gives the gateways flexibility to optimize beyond that possible using internetwork dalagrams where packet boundaries must be preserved by gateways.

A TCP module is included in the gateway to allow it to establish connections to remote gateways for communication with remote processes corresponding to the alias processes it implements. This ICP implementation is also made available as a service by the gatcway to clients on the local network requiring internetwork RlA/IT. Thus, a program can use TCP connections as though TCP was implemented on their workstalion even though it is in fact implemented in the gateway and no $\mathrm{IP} / \mathrm{TCP}$ is used on the local network. Similar modules could be provided in the gateway for other internetwork protocols.
In summary, our gateway provides a full range of services for internetwork communication ranging from access control to optimization on long-haul communication to implementation of internetworking protocols. 'This functionality localizes the cost of internetworking to the gateway and protects the autonomy of the local network as an administration, protection and security domain.

\section{Current Status}

The $\mathrm{V}$ kernel has been in use with diskless workstations at Stanford and elsewhere for more than a year. In the diskless configuration, the kerncl and a command interpreter are resident on the workstation. A variety of applications run in this environment, including a VLSI layout editor, interactive debugger, two text editors, illustrators and various standard command facilitics. An initial form of remote command exccution is in use. File access is currently provided by server programs on our VAX/UNIX systems. A server-based TCP implementation has been in use for one year as well.

Our initial gateway is targeted to connect 3 megabit and 10 megabit Ethernet since we are in the process of switching to 10 megabit Ethernet. At the time of writing, the gateway code for handling the Send-Receive-Reply sequence, crcation of alias processes and use of TCP connections is written and passes initial tests, but only in single processor configuration. We hope to have the gateway in production use later this ycar.

\section{Concluding Remarks}

Local networks provide cheap and powerful communication yet pose some hard problems, like performance, reliable operation and security. The distributed V-System achieves high performance on the local network with a transparent network IPC facility based on a simple request-response interkernel protocol. Mcasurements indicate that this facility gives performance superior to internetwork protocols and the performance is sensitive to additional protocol overhead. In particular, processor time is the critical factor

Several mechanisms have been described for internetworking none of which imposes a penaity for local network performance. We argue that the V-System design is a practical, efficient approach that both builds on the current work and standards for internetworking and also provides a possible base for local networks standardization.

The novel aspects of our system are:

- Transparent, message-based interprocess communication for all transport-level local network communication as well as intra-host communication.

- Use of a request-response type of transport protocol for local network communication.

- The local network as a protection, security and reliability domain with the required policies implemented by intelligent gateways structured as two connected half gateways.

- Semi-transparent internetwork operation of the interprocess communication at no penalty to local network performance.

The interprocess communication facility suffices as the single transport-level communication mechanism on the loca! network as well as for all inter-program communication, whethar local or distributed. It is as efficient to use for local network file access, file transfer and terminal access as more specialized protocols.

The use of intelligent gateways that support and control transport-level communication into and out of a local network protects the typically failure-prone and unprotected local network from the internetwork and insulates the local network protocol and its performance from internetwork considerations.

The use of a single transport protocol on the local network 
minimizes the the protocol-translation problems for the gateway. The active role of a gateway in protocol translation provides a good basis for imposing access control policies between a local network and the internetwork.

\section{Acknowledgements}

Willy Zwacnepocl and Tim Mann are major contributors to the $V$ kernel implementation. Domingo Lampaya has contributed to the refinement of these ideas and the implementation of the V-System gatcway. The Internet server providing IP/TCP in the gateway was implemented by Marvin Theimer. These colleagues have also made valuable comments and suggestions on this paper.

\section{References}

1. D.R. Cheriton and W. Zwaenpoel, "The Distributed V Kernel and its Performance for Diskless Workstations," Proceedings of the 9th Symposium on Operating System Principles, ACM, 1983, .

2. Digital Equipment Corporation, Intel Corporation and Xerox Corporation, "The Ethernet: A Local Area Network - Data Link I ayer and Physical Layer Specifications, Version 1.0",

3. A. Bechtolsheim, F. Baskett, V. Pratt, "The SUN Workstation Architecture," Tech. report, Computer Science Department, Stanford University, January 1982.

4. M.V. Wilkes and D.J. Wheeler, "The Cambridge Digital Communication Ring," Local Area Communication Networks Symposium, Mitre Corp. and National Burcau of Standards, 1979, .

5. J. Saltzer and K. Pogran, "A Star-shaped Ring Network with High Maintainability," Proc. Local Area Communication Network Symposium, May 1979, .

6. B.J. Nelson, Remote Procedure Call, PhD dissertation, Carnegie-Mellon U., 1981, published as CMU technical report CMU-CS-81-119

7. A. Spector, "Performing Remote Operations Efficiently on a Local Computer Network," Comm. ACM, Vol. 25, No. 4, April 1982, pp. 246-260.

8. A.D. Birrell and B.J. Nelson, "Communication Techniques for Remote Procedure Calls," Proceedings of the 9th Symposium on Operating System Principles, ACM, 1983,.

9. J.B: Postel, "Internctwork Protocol Approaches," IEEE Trans. on Comm., April 1980, .

10. DARPA, "DOD Standard Internet Protocol," Tech. report IEN-128, Defence Advanced Research Projects Agency, January 1980.

11. DARPA, "DOD Standard Transmission Control Protocol," Tech. report IEN-129, Defence Advanced Research Projects Agency, January 1980.

12. D.R. Boggs, J.F Shoch, E.A. Taft and R.M Metcalfe, "Pup: An Internetwork Architecture," IEEE Trans. on Comm., April 1980, .

13. D. Clark, "IP Datagram Reassembly Algorithms," Tech. report RFC 815, DARPA, 1982.

14. D. Clark, "Modularity and Efficiency in Protocol Implementation," Tech. report RFC 817, DARPA, 1982.

15. G. Popek, B. Walker, J. Chow, D. Edwards, C. Kline, G.Rudisin, G. Thiel, "LOCUS: A Network Transparent, Iligh Reliability Distributed System," Proceedings of the 8th Symposium on Operating Systems Principles, ACM, December 1981, pp. 169-177. 